\title{
Baetis ingridae n. sp., Ephéméroptère nouveau de Corse (Baetidae)
}

\author{
A.G.B. Thomas 1 \\ T. Soldán ${ }^{2}$
}

Mots clés : Ephemeroptera, Baetidae, nouvelle espèce, Corse.

Description illustrée de la larve au dernier stade de Baetis ingridae n. sp., rencontrée en Corse. Cette espèce appartient au groupe rhodani.

Baetis ingridae n. sp., a new mayfly from Corsica (Baetldae).

Keywords : Ephemeroptera, Baetidae, new species, Corsica.

An illustrated description of the last-instar larva of Baetis ingridae n. sp., found in Corsica. This species belongs to the rhodani group.

\section{Introduction}

Depuis fort longtemps, une espèce identifíe comme Chloe "Rhodani? "Pictet (sic)a été signalée de Corse (Hagen, I864). L'auteur a ajouté n'avoir pu, à l'époque, comparer ses spécimens à ceux de la collection Pictet. Kimmins (1930) a repris cette identification douteuse, en modifiant seulement la combinaison binominale ( ? Baetis thodani Pict.). La citation de Baetis rhodani par Lestage (1922), recopiée ${ }^{3}$ par Verrier (1954) puis d'ąutres auteurs, est très probablement erronée.

En effet, la prospection récente de divers cours d'eau de Corse par plusieurs chercheurs a permis l'identification d'une espèce nouvelle qui paraît vicariante de $B$. rhodani et que nous sommes heureux de dédier au Dr Ingrid Müller-Liebenau (Plön).

1. Laboratoire d'Hydrobiologie, UA 695 C.N.R.S. Université Paul Sabatier, 118, route de Narbonne, 31062 Toulouse Cedex, France. 2. Ceskoslovenska akademie ved, Entomologicky ústav, Branisovska 31, 370 o5 Ceske Budejovice, Tchécoslovaquie.

3. M.L. Verrier n'a pas identifié de larves de $B$. rhodani fou du groupe rhodani) dans la collection E. Angelier qui lui avait été confiée pour étude.

\section{Baetis ingridae $n$. sp. : description}

\section{LARVE AU DERNIER STADE}

Diagnose sommaire : espèce de taille moyenne à petite, typiquement du groupe rhodani, avec de fortes épines marginales sur les branchies et de nombreuses écailles bordantes distales sur les tergites abdominaux. Coloration d'ensemble relativement claire et modérément contrastée. Paracerque de longueur assez voisine de la moitié de celle des cerques.

Tête

Antennes : scape et surtout pédicelle (fig. 1) porteurs de fortes écailles très arrondies à l'apex.

Labre (fig. 2). Nombre de grandes soies frontales : $1+5-10$, le plus souvent 7 ou 8 , soit un peu moins que chez B. rhodani (en général $1+7-12$ : voir Müller-Liebenau, 1969, p. 95).

Mandibules (fig. 3), à fortes denticulations et à prosthecas nettement asymétriques et bien développées.

Maxilles : palpes maxillaires à apex (fig. 4) légèrement tronconique, portant une denticulation bien visible et de fines soies. 


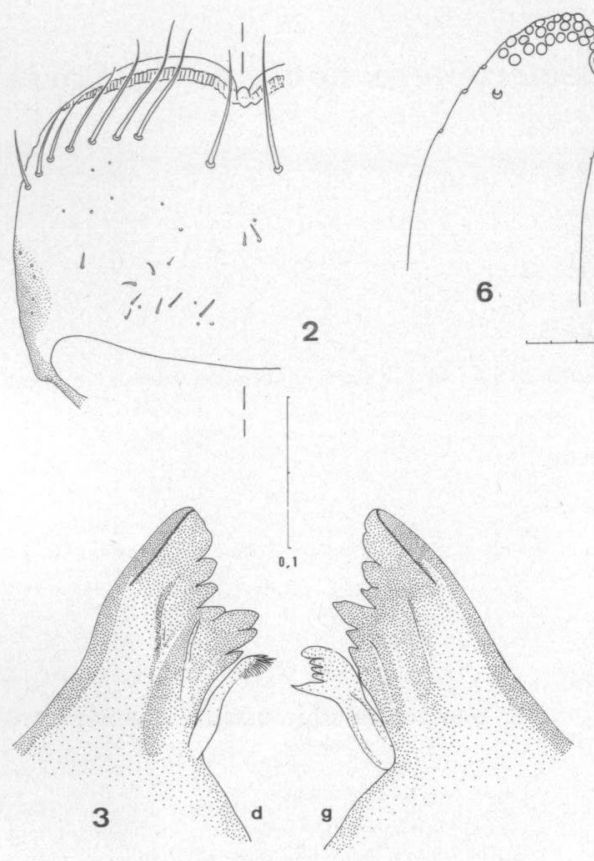

0,03

4
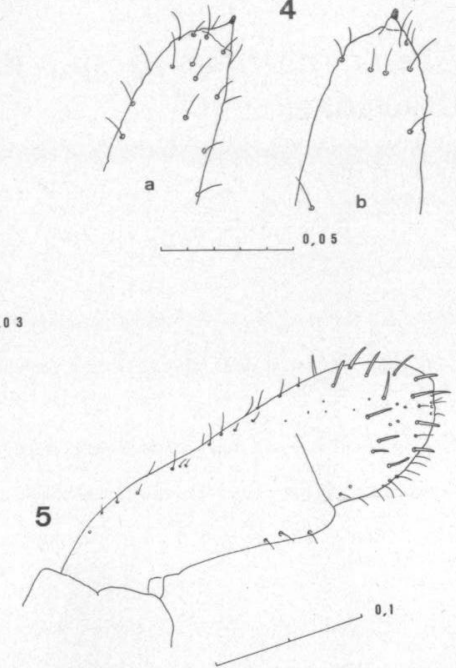

9

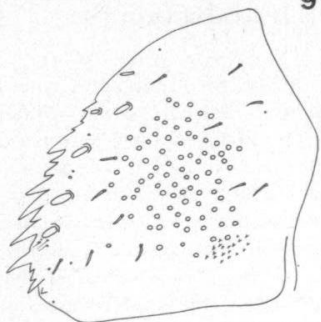

8
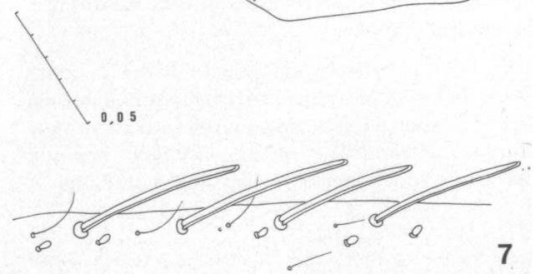

Fig. 1 à 9. Larves de Baetis ingridae $\mathbf{n}$. sp. au dernier stade. Echelle en $\mathrm{mm} .1$ : pédicelle. $2:$ labre. 3 : apex et prostheca des mandibules droite (d) et gauche (g). $4 a$ et $b$ : apex du palpe maxillaire chez deux individus. $5: 2^{c}$ et $3^{c}$ articles du palpe labial, vue ventrale. 6 : paraglosse, vue ventrale 7 : bord postérieur du fémur, région moyenne. $8: 4$ e branchie, bord antéro-externe. 9 : paraprocte. 


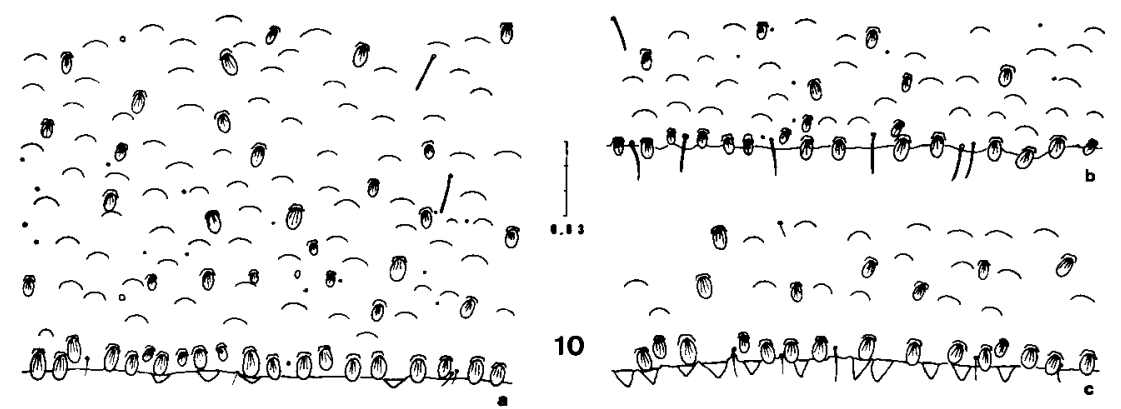

Fig. 10. Larve de Baetis ingridae n. sp. au dernier stade. Echelle en $\mathrm{mm}$. Bord postérieur et surface du $4^{e}$ tergite dans la région distale médiane (a). Bords postérieurs du $2^{e}$ tergite (b) et du $7^{e}$ (c).

Labium : palpes à ensemble des articles $2+3$ étroit et allongé (rapport longueur/largeur de l'ordre de 2,7 à 3,0 , soit beaucoup plus que chez $B$. rhodani où il est voisin de 2), avec un faible dépassement interne du second article (fig. 5).

Paraglosse : apex assez arrondi avec un nombre de soies modéré (fig. 6).

\section{Thorax}

Pronotum à maculations proches de celles de $B$. rhodani, dans l'ensemble.

\section{Pattes}

Bord postérieur des fémurs frangé de fortes soies, net tement arrondies à l'extrémité (fig. 7). Griffes tarsales puissantes, fortement recourbées et dépourvues de soies subapicales sur toutes les pattes examinées.

\section{Abdomen}

Ensemble plutôt clair. Coloration dorsale sensiblement plus foncée sur les segments 3,6 et 7 . Segment 2 avec deux petites taches brun foncé de part et d'autre du plan de symétrie. Une tache médiane claire longitudinale (plus ou moins élargie sur les tergites 4-5) est en général visible du $3^{\mathbf{e}}$ au $7^{\mathbf{e}}$ - parfois $8^{e}$ ou même $9 e$ - segments.

La surface des tergites porte de nombreuses impressions concaves très marquées et de fortes écailles arrondies. Quelques formations anguleuses à base convexe sur la partie proximale des tergites, souvent assez peu visibles. Bord postérieur des tergites constitué d'écailles en surplomb et de larges denticulations, rares ou absentes sur les premiers tergites, et de plus en plus abondantes vers l'arrière de l'abdomen ( $f$ ig. $10 \mathrm{a}$ à c).

Branchies à liséré brun et à réseau trachéen très pigmenté. Bord antéro-externe porteur de fortes épines de type rhodani (fig. 8).

Paraproctes (fig. 9) : bord postéro-interne armé d'une dizaine de fortes épines. Quelques écailles sous-marginales et des soies peu nombreuses. Par contre, des traces circulaires en assez grand nom. bre confèrent à la région centrale de la paraprocte un aspect de " crible ", bien visible à faible grossis. sernent.

Cerques : brun clair; longueur du paracerque comprise entre 0,4 et 0,65 fois celle des cerques.

\section{Taille}

Elle varie dans de larges limites, cas fréquent en montagne, mais dans l'ensemble elle est inférieure à celle de $B$. rhodani.

Longueurs $(\mathrm{mm})$ :

- du corps : 4,2 à $8,3(\sigma), 4,6$ à $8,8(8)$;

- des cerques: 3,7 à $5,8(\sigma), 3,7$ à $6,3(Q)$;

- du paracerque $: 1,7$ à $3,0(\infty), 1,9$ à $2,9(\varphi)$. 


\section{Matériel examiné}

31 larves au dernier stade (21 o et 10 \&) provenant de :

Le Bevinco à $280 \mathrm{~m}, 9$ - VII - 1985 (M. Sartori leg.); le Golo à $330 \mathrm{~m}, 6$ - VII - 1985 (M. Sartori leg.) et son affluent à $970 \mathrm{~m}$ dans la forêt de Valdo Niello (ibid.); la Restonica vers $1150 \mathrm{~m}, 7$ - VIII - 1983 (G. Gazagnes leg.) et de 400 à $600 \mathrm{~m}$. VI - 1983 (J. Giudicelli leg.) ; le ruisseau en aval de la cascade du Voile de la Mariè à $950 \mathrm{~m}$, sur le versant Sud du col de Vizzavona, 5 - VII - 1985 (M. Sartori leg.); le Taravo a $240 \mathrm{~m}, 7$ - VII - 1985 (M. Sartori leg.) ; le Rizzanese à $50 \mathrm{~m}, 7$ - VII - 1985 (M. Sartori leg.). Holotype : une larve \& provenant du Bevinco a $280 \mathrm{~m}, 9$ - VII - 1985.

4 spécimens sont déposés au Musée Zoologique de Lausanne, 4 autres dans la collection Soldán à Ceské Budejovice, le reste dans la collection Thomas à Toulouse.

\section{Remerciements}

C'est un plaisir de remercier amicalement pour le legs de leur matériel : M. et Mme G. Gazagnes (Toulouse), M. J. Giudicelli (Mar* seille) et M. M. Sartori (Lausanne).

Travaux cités

Hagen (H.A.). 1804. - Nevropteres (non Odonates) de la Corse, recueillis par M.E. Bellier de la Chavigmerie en 1860 et 1861 . Annls Soc. ent. Fr., $4: 38-45$.

Kimmins (D.E.). 1930. - A list of the corsican Ephemeroptera and Neuroptera. Eos, $6:$ 185-190.

Lestage (J.A.). 1922. - Catalogue des Ephémères de France. Anmis Soc. ent. Fr., $91: 273-276$.

Müller-Liebenau (I.). 1969. - Revision der europäischen Arten der Gattung Baeris Leach, 1815 (Insecta, Ephemeroptera). Gewäss. A buäss., $48-49: 1-214$.

Verrier (M.L.). 1954. - Contribution à l'étude de la faune d'ean douce de Corse. Ephéméroptères. Vie Milieu, 5 (2) : 280-290. 